\title{
Measurement of temperature in a standard flame using multispectral thermography
}

\author{
by J. Meléndez *, J. Talavante *, G. Guarnizo *, and M. J. Martín** \\ * Physics Department, Universidad Carlos III de Madrid, Leganés, Spain, melendez@fis.uc3m.es \\ ** Centro Español de Metrología, Tres Cantos, Spain, mjmartinh@cem.es
}

\begin{abstract}
Accurate measurement of temperature in combustion processes is a challenging metrological problem with important practical applications. This paper describes an innovative measurement method of temperature in a standard flame using a multispectral camera with six spectral bands in the medium infrared. The obtained results are compared to measurements with Rayleigh scattering thermometry, a technique traceable to the International Temperature Scale of 1990 (ITS-90), and with temperature maps obtained with a hyperspectral imager. The proposed method provides also measurement of the column density $(\mathrm{ppm} \cdot \mathrm{m})$ of $\mathrm{CO}_{2}$ in the flame.
\end{abstract}

\section{Introduction}

Accurate measurement of the temperature of combustion processes can significantly improve their efficiency and reduce harmful emissions. State-of-the-art optical diagnostics are non-intrusive, and thus preferable to thermocouples, but they are generally complex laboratory techniques with uncertainties between $5 \%$ and $10 \%$ of the process temperature. One of the goals of the European Metrology Programme for Innovation and Research (EMPIR) project EMPRESS2 (which has funded this work) is to achieve $\approx 0.5 \%$ uncertainty with an imaging system, based on a multispectral camera in the medium infrared (MIR), simple and rugged enough to be used in industrial environments.

\section{Hyperspectral thermography of flames}

Thermography of flames is complicated by the strong spectral structure of emissivity and its dependence on temperature $(T)$ and species column density $(Q$, concentration times optical path). However, these dependences are well known for the main chemical species, so that for a couple of $(T, Q)$ values, the spectral emissivity and thus the spectral radiance can be calculated on a line-by-line basis using the HITRAN database [1]. Then, these calculated emission spectra can be used to fit iteratively a measured spectra, the best fit providing a retrieved $(T, Q)$ value. This procedure has been applied, for the spectral region of $\mathrm{CO}_{2}$ emission, to spectra acquired by an FTIR hyperspectral imager with 0.5 $\mathrm{cm}^{-1}$ spectral resolution, and has demonstrated its ability to measure temperature in a standard flame (T₹2000 K) to an estimated uncertainty of $\pm 5 \mathrm{~K}$ (i.e., $\approx 0.25 \%$ ) [2].

\section{Multispectral thermography of flames}

Measurement of high resolution emission spectra for a whole image requires expensive instrumentation, long acquisition times and very large data files. However, values of $T$ and $Q$ do not only affect the fine structure of the spectra, but also the overall shape (see Figure 1), and therefore it seems possible to retrieve them from low resolution measurements.
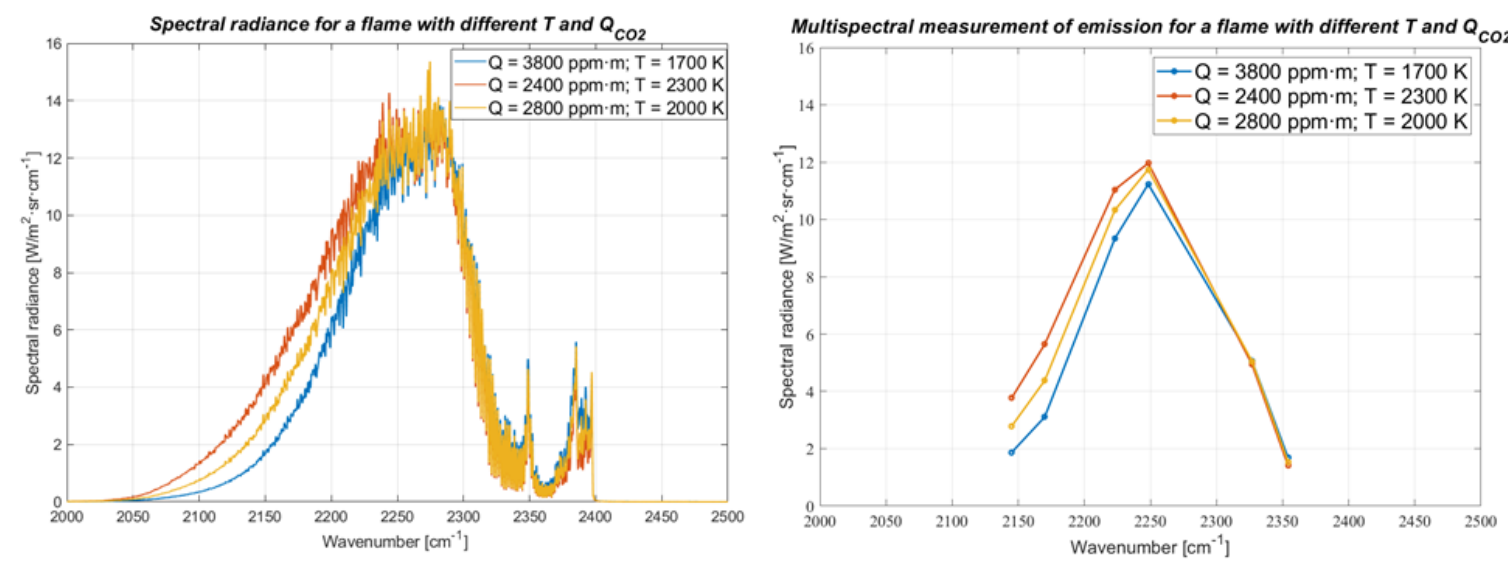

Fig. 1. (Left) Simulated spectra for three different values of $T, Q_{\mathrm{CO} 2}$. (Right) Simulated multispectral measurement with the filters in Table I for the same values of $T$ and $Q_{c o 2}$. 
In this work, a MIR camera (3 to $5 \mu \mathrm{m}$ ) provided with a rotating filter wheel has been used to obtain multispectral (6-band) images of the same standard flame previously studied by hyperspectral spectroscopy and Rayleigh scattering thermometry. The camera has been radiometrically calibrated in each band with high temperature cavity blackbodies at Centro Español de Metrología (CEM). The filters used are detailed in Table 1.

Table 1. Center wavelengths and full widths at half maximum transmission (FWHM) of the filters.

\begin{tabular}{|l|c|c|c|c|c|c|}
\hline Filter & A1 & A2 & A3 & A4 & A5 & A6 \\
\hline Center $\lambda(\mathrm{nm})$ & 4500 & 4665 & 4450 & 4610 & 4300 & 4250 \\
\hline FWHM $(\mathrm{nm})$ & 170 & 240 & 200 & 175 & 200 & 190 \\
\hline
\end{tabular}

\subsection{Measurement method}

High resolution emission spectra have been calculated using the HITRAN database, for a wide range of $T$ and $Q_{C O 2}$ values. These spectra have been integrated over each of the chosen multispectral bands to simulate camera measurements. For each pixel of the image, the set of six measured radiances is compared to the simulated ones, for all the $(T, Q)$ couples. The values of temperature and $\mathrm{CO}_{2}$ column densities assigned to the pixel are those that minimize the sum of absolute errors. It must be stressed that simulated spectra have been calculated assuming a flat profile of $(T, Q)$, and therefore the retrieved values must be understood as a line-of-sight effective average of the flame temperature. However, temperature profiles in the standard flame measured are very flat, and systematic errors due to spatial nonuniformity of temperature have been estimated to be smaller than $15 \mathrm{~K}$ [2].

\subsection{Results}

Figure 2 shows the temperature maps obtained with the multispectral (left) compared to the hyperspectral (right) measurements. Qualitative agreement is very good, although multispectral $T$ values in the lower central zone (the hottest region of the flame and the most uniform spatially) are about $100 \mathrm{~K}$ higher than hyperspectral $T$ ( $\approx+5 \%$ error). Multispectral maps of $Q$ (not pictured) have values about $400 \mathrm{ppm} \cdot \mathrm{m}$ lower than hyperspectral $Q(\approx-11 \%$ error). It can be concluded that multispectral thermography of flames is feasible, with accuracy for the standard flame studied comparable to that of optical diagnostics much more complex and expensive.

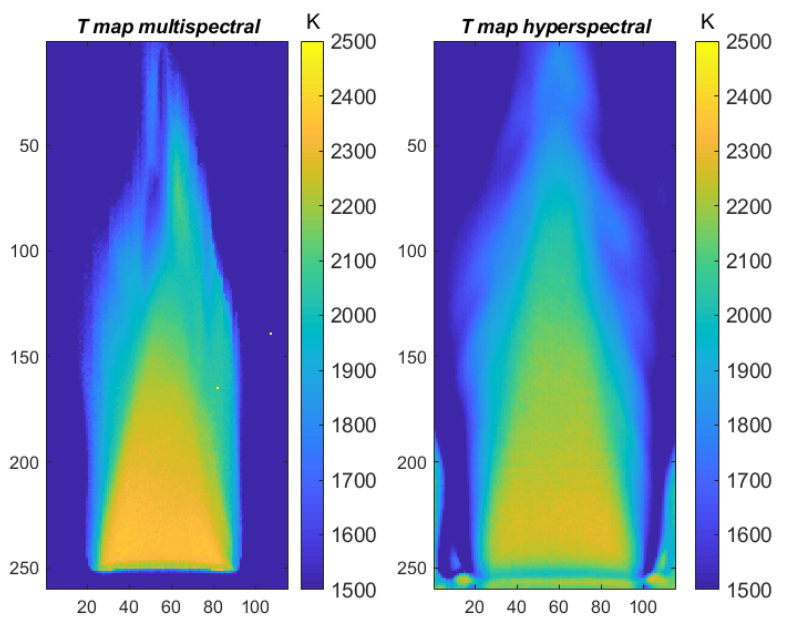

Fig. 2. Temperature maps for the standard flame obtained from multispectral (left) and hyperspectral (right) measurements

\section{REFERENCES}

[1] L. S. Rothman et al. "The HITRAN 2008 molecular spectroscopic database," J. Quant. Spectrosc. Radiat. Transfer 110, 533-572 (2009).

[2] Sutton, G., Fateev, A., Rodríguez-Conejo, M.A. et al. "Validation of Emission Spectroscopy Gas Temperature Measurements Using a Standard Flame Traceable to the International Temperature Scale of 1990 (ITS-90)". Int $J$ Thermophys 40, 99 (2019) doi:10.1007/s10765-019-2557-6 